\title{
Experimental study for the conditions of analog switching in ferroelectric liquid crystal cells
}

\author{
V. Manjuladevi, Yu. P. Panarin, ${ }^{\text {a) }}$ and J. K. Vij ${ }^{\text {b) }}$ \\ Department of Electronic Engineering, Trinity College Dublin, University of Dublin, Dublin 2, Ireland
}

(Received 17 April 2007; accepted 9 July 2007; published online 3 August 2007)

\begin{abstract}
The authors study the effect of the spontaneous polarization $\left(P_{S}\right)$ of a ferroelectric liquid crystal mixture with compensated helix and of the thickness of the alignment layer $\left(d_{a}\right)$ on the analog switching in a cell. The quality of analog switching is established in terms of its contrast ratio, texture in the dark state, and the electrostatic energy. The latter approximates to $P_{S}^{2} d_{a}$ for $d_{a} \ll d$ (thickness of the ferroelectric liquid crystal cell layer). () 2007 American Institute of Physics. [DOI: $10.1063 / 1.2767210]$
\end{abstract}

The discovery of the electro-optic effect called surface stabilized ferroelectric liquid crystal cell ${ }^{1}$ (SSFLC) led to low-cost passive-matrix wide viewing angle displays as attractive alternatives to that of nematics. Though a number of FLCs were designed and the addressing schemes advanced over a decade ago, a large-scale production of such microdisplays has only recently begun. ${ }^{2}$ The limiting reasons have been (i) a significant drop in the cost of thin film transistor technology and (ii) an absence of the analog gray scale in FLCs. However, the discovery of the electro-optic effect, thresholdless switching ${ }^{3}$ also called "V-shaped switching" in SSFLCs, has added attraction to potential for such displays in projections, viewfinders, and the switching devices using R-G-B color sequential scanning due to gray scale and the fast switching. This effect shows smooth, domain-free dependence of the transmission on applied voltage.

Although a number of V-shaped FLC displays have been prototyped and developed, ${ }^{4-6}$ the physical interpretation of the relevant electro-optic effect has been a topic of debate over a decade. Originally, the V-shaped switching achieved in the "Tokyo mixture" was explained as due to frustration between ferro- and antiferroelectricities, ${ }^{3}$ due to a ferrielectric phase ${ }^{7}$ with $q_{T}=2 / 3$, and biaxial anchoring. ${ }^{8}$ Nevertheless, V-shaped switching was also achieved in conventional FLCs which offered a differing interpretation. Several theoretical models ${ }^{9-13}$ sometimes controversial have been proposed to simulate conditions for observing this effect. One of the reasons for this controversy is the term "V shape." This is based on a particular observation of a smooth dependence of the transmittance on applied voltage. Similar response is also known for other FLC structures: short-pitch helical cells, ${ }^{14}$ chevron, and de Vries smectics. ${ }^{15}$ The conditions required for analog switching have not been clearly established though the importance of certain parameters such as (a) the large spontaneous polarization, (b) thick alignment layers, (c) relatively long (or compensated) helical pitch, (d) absence of domains during switching, and (e) existence of hysteresis inverse frequency have been known. A combination of the requirements, however, allows us to "localize" the term V shape and assign it to a nonuniform splayed structure. Such a structure might be realized with a combination of certain conditions, e.g., a large $\gamma_{p} / \gamma_{d}$ ratio, ${ }^{11,16}$ where $\gamma_{p}$ and $\gamma_{d}$ are

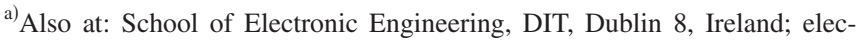
tronic mail: yuri.panarin@dit.ie

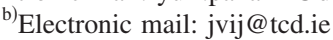

the polar and the nonpolar parts of the anchoring energy. The effect of large $\gamma_{p} / \gamma_{d}$ is increased by high $P_{S}$ values and the use of thick alignment layers ${ }^{17} d_{a}$. Moreover the splay structure is formed exclusively by large $P_{S}$ and thick $d_{a}$. A preliminary experimental study showed ${ }^{18}$ that the originally bistable cells became thresholdless (or monostable) with an increase in $d_{a}$ up to $1 \mu \mathrm{m}$ and the conventional hysteresis at moderate frequencies (i.e., $f>1 \mathrm{~Hz}$ ) inversed at frequencies lower than the hysteresis-inverse frequency $f_{i}$. According to the "electrostatic" model used here, $P_{S}$ creates an internal electric field which is proportional to both $P_{S}$ and $d_{a}$. This internal field interacts with the spontaneous polarization making the uniform (up and down) states unstable. Therefore, for sufficient large $P_{S}$ and $d_{a}$ the only stable state in the cell is that of the splayed structure, because the spontaneous polarization vectors are opposite thus canceling out with each other. The internal field consequently is zero in the absence of the external field. Recently O'Callaghan ${ }^{19}$ showed quantitatively that $\mathrm{V}$-shaped switching occurs when the electrostatic energy in the cell sufficiently exceeds surface anchoring energies, the latter expected to be of the order of $1 \mathrm{erg} / \mathrm{cm}^{2}$.

In this letter, we provide an evidence for the conditions of V-shaped switching in SSFLCs while examining the electrostatic model. According to this model, different parameters $\left(P_{S}, d_{a}\right.$, pitch, and anchoring energy) account for $\mathrm{V}$-shaped structure/switching. Here we vary $P_{S}$ and $d_{a}$ independently of each other. In our study a series of FLC mixtures with $P_{S}$ ranging from 24 to $96 \mathrm{nC} / \mathrm{cm}^{2}$ and compensated helix $\left(p_{0} \rightarrow \infty\right)$ are designed. A second set of the experiments is designed to study the effect of the thickness of the alignment layers; these are varied from 40 to $400 \mathrm{~nm}$. We address a number of problems including the term V-shape switching. The FLC mixtures used in the study show simple phase transition sequences (Is-SmA-SmC ${ }^{*}-\mathrm{Cr}$ ). This rules out other possible physical mechanisms for the V-shape switching such as de Vries ${ }^{15}$ and ferri- and/or antiferroelectric phases. ${ }^{3,7}$ The helical pitch of the studied mixture is rather long compared to the cell thickness to guarantee the absence of the helix and consequently the deformed helix ferroelectric (DHF) mode ${ }^{14}$ is ruled out as the reason for switching. Only two FLC structures may be present in these conditions: chevron structure and electrostatically stabilized splay (twist) structure. ${ }^{9-11}$ The transition from chevron to the monostable splay structure may occur at some critical value of the electrostatic energy depending on 
both $P_{S}$ and $d_{a}$. In practice, however, this transition is broad and has been discussed in detail ${ }^{16}$ where they find that for $P_{S}>100 \mathrm{nC} / \mathrm{cm}^{2}$, the effect of chevron is negligible with a consequential transition to the monostable state. Therefore it is important to introduce a quantitative parameter characterizing the quality of V-shape switching. Normally the extinction angles of up and down states in the chevron cell are rather low $\left(3^{\circ}-5^{\circ}\right)$ thus the electro-optical response may though appear similar to $\mathrm{V}$ shape at zero applied voltage, the chevron cell consists of (ideally 50/50\%) mixtures of up and down domains and the overall structure must show lower extinction than the monostable splay cell. Therefore in our study we utilized the contrast ratio (CR) as a qualitative parameter for characterizing the quality of the V-shape switching and transition from chevron to splayed structures. The second, rather useful measure of the V-shape quality is just the texture of the cell at zero applied voltage obtained using a polarizing microscope.

We prepared a series of FLC mixtures with compensated helix (helix free) with $P_{S}$ varying from $\sim 96$ to $24 \mathrm{nC} / \mathrm{cm}^{2}$ by varying the concentration of two optically active dopants, with the same sign of $P_{S}$ but opposite signs of the helical pitch $p_{0}$. For electro-optical studies, planar cells were prepared using the indium tin oxide coated glass plates and polyimide RN1175 as the alignment layer. The alignment layer thickness was varied from 40 to $400 \mathrm{~nm}$ using the spin coating procedure. One side rubbed cells with thickness in the range of 1.4-1.8 $\mu \mathrm{m}$ were prepared. The cell was filled with the material in the isotropic phase and then cooled down to room temperature. The rubbing direction of the cell substrate was adjusted parallel to one of the crossed polarizers of the microscope. A triangular voltage was applied at various frequencies and the transmittance measured using a photodiode attached to the microscope. The electro-optic response at various frequencies was recorded using an oscilloscope. The images in the dark state were recorded using a digital camera interfaced with the computer.

The effect of the spontaneous polarization on the appearance of the $\mathrm{V}$-shaped switching was studied by measuring the electro-optic response of the FLC mixtures with different $P_{S}$. The helical pitch of the mixtures remains rather long $(>10 \mu \mathrm{m})$ to guarantee the surface stabilized helix-free structure. The smectic tilt angle at room temperature of all the mixtures lies in the range of $26^{\circ}-27^{\circ}$. The electro-optic response of the material with $P_{S}=96 \mathrm{nC} / \mathrm{cm}^{2}$ with various thicknesses of the alignment layer are shown in Fig. 1(a). Figure 1(b) shows the electro-optic response of the various cells with FLC mixtures with $P_{S}$ ranging from 96 to $24 \mathrm{nC} / \mathrm{cm}^{2}$ with $d_{a} \sim 400 \mathrm{~nm}$. The response is V-shaped switching at a frequency of $3 \mathrm{~Hz}$ in cells with $d_{a}$ ranging from 200 to $400 \mathrm{~nm}$. The texture is uniformly black with a single domain as seen in Fig. 2(a). Though the response of the cell with $d_{a}$ of $200 \mathrm{~nm}$ looks V-shaped, the CR drops from 92 to 33 due to the stripe-shaped domains appearing in the texture in Fig. 2(b). V-shaped switching is no (a)
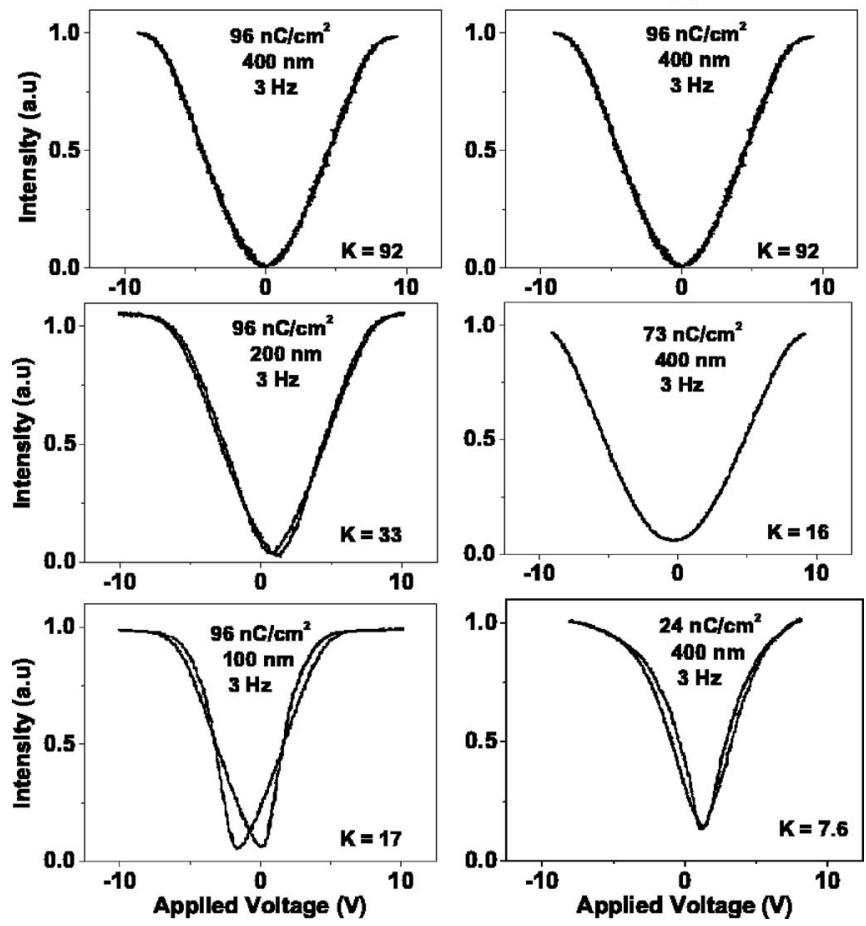

FIG. 1. Electro-optic response of FLC mixture: (a) $P_{S}=96 \mathrm{nC} / \mathrm{cm}^{2}$ and varying thickness of alignment layers; and (b) $d_{a} \sim 400 \mathrm{~nm}$ and varying $P_{S}$. $\mathbf{K}$ here is the contrast ratio.

longer observed for $d_{a} \sim 100 \mathrm{~nm}$ and below. With a change in $P_{S}$ from 96 to $73 \mathrm{nC} / \mathrm{cm}^{2}$ the response remains V-shapedlike but the CR drops again to only 16 due to the presence of domains, as shown in Fig. 2(c). Finally, for the FLC cell with $24 \mathrm{nC} / \mathrm{cm}^{2}$ the response is no longer $\mathrm{V}$ shaped.

Figures 3(a) and 3(b) show the dependence of the hysteresis width (or coercive voltage) as a function of frequency. At higher frequencies the electro-optic response of all cells shows positive hysteresis due to the dynamic delay in switching. At low frequencies, the electro-optic response shows negative or inversed hysteresis due to a difference between the applied and internal electric fields. ${ }^{15,17,18}$ The internal (or actual) electric field $E(t)$ inside a FLC cell is a sum of the field due to the applied voltage and the field due to surface charges distributed between the two electrodes of the cell and can be written as ${ }^{17}$

$$
E(t)=-\frac{V(t) \varepsilon_{a}}{2 d_{a} \varepsilon+d \varepsilon_{a}}+\frac{2 \sigma(t) d_{a}}{2 d_{a} \varepsilon+d \varepsilon_{a}},
$$

where $V(t)$ is the voltage of the triangular wave applied across the FLC cell, $\varepsilon$ and $\varepsilon_{a}$ are dielectric permittivities of the LC and alignment layers, respectively, and $d$ and $d_{a}$ are the thicknesses of the LC cell and of the alignment layers, respectively. The sign in the electric field arises from this being the negative gradient of the potential function. $\sigma(t)$ is
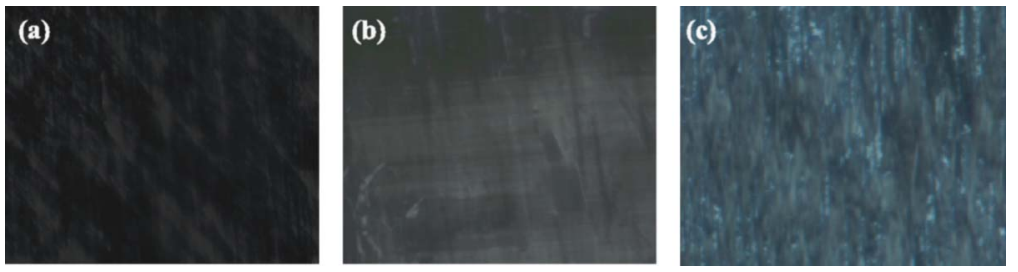

FIG. 2. (Color online) Photographs of the cell textures at zero applied field: (a) $P_{S}=96 \mathrm{nC} / \mathrm{cm}^{2}, d_{a} \sim 400 \mathrm{~nm}$; (b) $P_{S}=96 \mathrm{nC} / \mathrm{cm}^{2}, \quad d_{a} \sim 200 \mathrm{~nm} ;$ and (c) $P_{S}$ $=24 \mathrm{nC} / \mathrm{cm}^{2}, d_{a} \sim 400 \mathrm{~nm}$. The linear dimension of the texture image horizontally is $120 \mu \mathrm{m}$. 

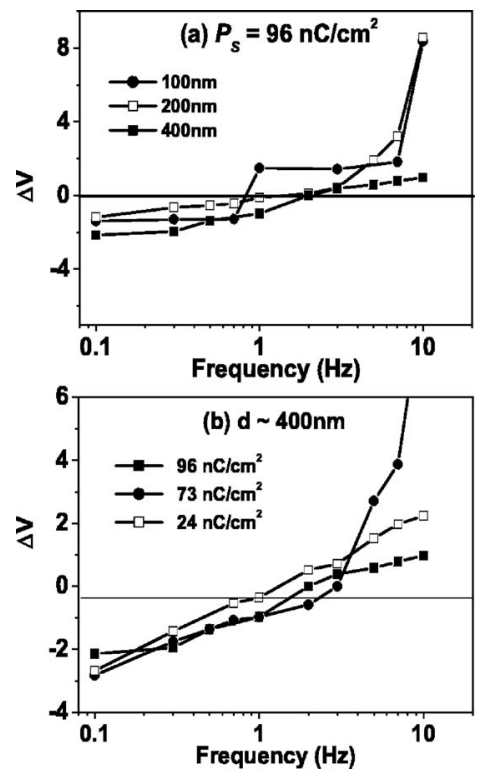

FIG. 3. Variation of coercive field in both cases (a) and (b) as a function of frequency.

the effective surface charge density that accumulates at the interface between the alignment layer and FLC as

$$
\sigma(t)=\sigma_{\text {ion }}+P_{s}\left(\cos \varphi_{1}+\cos \varphi_{2}\right),
$$

$\sigma_{\text {ion }}$ is the charge density due to the ions and the second term is due to the polarization charge density, $\varphi_{1}$ and $\varphi_{2}$ are the azimuthal angles of the directors measured with respect to the two aligning surfaces; these being zero for the directors lying along the aligning surfaces. The effect of ions on the appearance of the low frequency hysteresis has been examined. ${ }^{15,20}$ For higher frequencies, the field induced by the ions enhances the externally applied field, and at low frequencies it diminishes this field. Hence the internal field can be either greater or lower than the external field leading to a change in the coercive force. ${ }^{15}$ The frequency range of the hysteresis-free V-shaped switching can be increased considerably by connecting the FLC cell through the capacitor ${ }^{12}$ but in the case of pulse switching, the frequency is not of any significance. From the results presented in Figs. 1-3, we observe that the V-shaped switching is distorted by decreasing the CR on lowering either $P_{S}$ or by reducing $d_{a}$. These observations are explained in terms of the "electrostatic" model. ${ }^{9,10}$ Using Eq. (2), the electrostatic energy can be calculated. O'Callaghan ${ }^{19}$ discussed its importance and found it as $W_{0}=d_{a} P_{S}^{2} / \varepsilon_{a}\left(1+2 \varepsilon d_{a} / \varepsilon_{a} d\right)$. This approximates to $P_{S}^{2} d_{a} / \varepsilon_{\mathrm{a}}$ for $d_{a} \ll d$. In order to get $W_{0}$ in $\mathrm{erg} / \mathrm{cm}^{2}$, we need to multiply this expression by $4 \pi, \varepsilon$ and $\varepsilon_{a}$ are then dimensionless numbers, thickness and $P_{S}$ are in CGS units. At some critical value of the electrostatic energy $W_{0}^{*}$, it exceeds the elastic energy of the splay deformations and then the monostable (domainless) splay structure is realized. The CR is shown as a function of $W_{0}$ in Fig. 4 . The CR decreases by decreasing $W_{0}$ and for value $<4 \mathrm{erg} / \mathrm{cm}^{2}$, the CR is reduced due to the appearance of the switching (up and down) domains and the sample is no longer monostable.

The experimental results presented in this letter support the electrostatic, ${ }^{9-11}$ and related models ${ }^{17,18}$ according to which both $P_{S}$ and $d_{a}$ (thickness of the alignment layer) play important roles in giving V-shaped switching structure. We observe that this switching is distorted by lowering either $P_{s}$

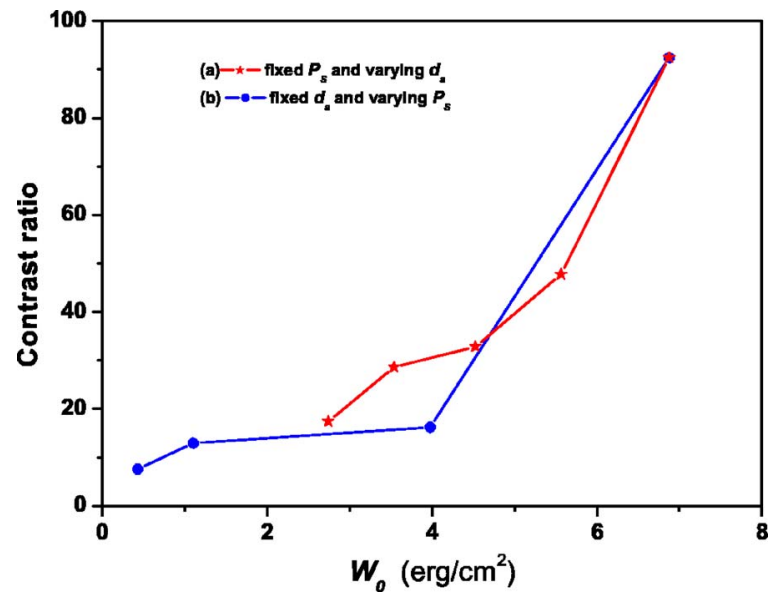

FIG. 4. (Color online) Contrast ratio as function of electrostatic energy $W_{0}$ for $\varepsilon=6$ and $\varepsilon_{a}=3$.

or $d_{a}$. The CR is reduced by lowering $W_{0}$ and for $W_{0}=W_{0}^{*}$ $<4 \mathrm{erg} / \mathrm{cm}^{2}$ due to the switching (up and down) domains appearing in the texture where the cell is no longer monostable. Therefore this value of $W_{0}^{*}$ is the critical value between the bistable and the monostable (V shaped) structures and it presumably exceeds the surface anchoring energy as predicted. ${ }^{19}$ It would also be important to study the effect of the helical pitch and of the cell thickness. These are the other two important parameters in addition to $P_{S}$ and $d_{a}$ for realizing a high-quality V-shaped switching.

The authors thank J. K. Song for useful discussions and Nissan Chemicals for gifting us RN1175.

${ }^{1}$ N. A. Clark and S. T. Lagerwall, Appl. Phys. Lett. 36, 899 (1980).

${ }^{2}$ http://www.displaytech.com/company.html

${ }^{3}$ S. Inui, N. Iimura, T. Suzuki, H. Iwane, K. Miyachi, Y. Takanishi, and A. Fukuda, J. Mater. Chem. 6, 671 (1996).

${ }^{4}$ T. Saishu, K. Takatoh, R. Iida, H. Nagata, and Y. Mori, SID Int. Symp. Digest Tech. Papers 1996, 703 .

${ }^{5}$ T. Yoshida, T. Tanaka, J. Ogura, H. Wakai, and H. Aoki, SID Int. Symp. Digest Tech. Papers 1997, 841.

${ }^{6}$ R. Hasegawa, H. Fujiwara, H. Nagata, Y. Hara, T. Saishu, R. Fukushima, M. Akiyama, H. Okumura, and K. Takatoh, J. Soc. Inf. Disp. 9, 107 (2001).

${ }^{7}$ M. Takeuchi, K. Chao, T. Ando, T. Matsumoto, A. Fukuda, and M. Yamashita, Ferroelectrics 246, 1 (2000).

${ }^{8}$ S. S. Seomun, J. K. Vij, N. Hayashi, T. Kato, and A. Fukuda, Appl. Phys. Lett. 79, 940 (2001).

${ }^{9}$ P. Rudquist, J. P. F. Lagerwall, M. Buivydas, F. Gouda, S. T. Lagerwall, N. A. Clark, J. E. Maclennan, R. Shao, D. A. Coleman, S. Bardon, T. Bellini, D. R. Link, G. Natale, M. A. Glaser, D. M. Walba, M. D. Wand, and X.-H. Chen, J. Mater. Chem. 9, 1257 (1999).

${ }^{10}$ N. A. Clark, D. Coleman, and J. E. Maclennan, Liq. Cryst. 27, 985 (2000).

${ }^{11}$ M. Copic, J. E. Maclennan, and N. A. Clark, Phys. Rev. E 65, 021708 (2002).

${ }^{12}$ L. M. Blinov, S. P. Palto, F. V. Podgornov, H. Moritake, and W. Haase, Liq. Cryst. 31, 61 (2004).

${ }^{13}$ S. Tzeng, T. Y. Lin, R. H. Haung, J. Wu, and S. Wu, Phys. Rev. E 70, 011712 (2004).

${ }^{14}$ B. I. Ostrovsky and V. G. Chigrinov, Kristallografiya 25, 560 (1980).

${ }^{15}$ Yu. P. Panarin, V. Panov, O. E. Kalinovskaya, and J. K. Vij, Ferroelectrics 246, 35 (2000).

${ }^{16}$ N. Vaupotič and M. Čopič, Phys. Rev. E 68, 061705 (2003).

${ }^{17}$ K. H. Yang, A. Lien, and T. C. Chieu, Jpn. J. Appl. Phys. 27, 2022 (1988).

${ }^{18}$ Yu. P. Panarin, Ph.D. thesis, Organic Intermediates Dyes Institute, Moscow, 1993; Yu. P. Panarin, Mols. Mats. 2, 137 (1993).

${ }^{19}$ M. J. O'Callaghan, Phys. Rev. E 67, 011710 (2003).

${ }^{20}$ M. Čopič, J. E. Maclennan, and N. A. Clark, Phys. Rev. E 63, 031703 (2001). 